\title{
Impact of Polar Edge Terminations of the Transition Metal Dichalcogenide Monolayers during Vapor Growth
}

Deng, Qingming; Thi, Quoc Huy; Zhao, Jiong; Yun, Seok Joon; Kim, Hyun; Chen, Guibin; Ly, Thuc Hue

Published in:

The Journal of Physical Chemistry Part C

Link to article, DOI:

10.1021/acs.jpcc.7b09332

Publication date:

2018

Document Version

Peer reviewed version

Link back to DTU Orbit

Citation (APA):

Deng, Q., Thi, Q. H., Zhao, J., Yun, S. J., Kim, H., Chen, G., \& Ly, T. H. (2018). Impact of Polar Edge

Terminations of the Transition Metal Dichalcogenide Monolayers during Vapor Growth. The Journal of Physical Chemistry Part C, 122(6), 3575-3581. https://doi.org/10.1021/acs.jpcc.7b09332

\section{General rights}

Copyright and moral rights for the publications made accessible in the public portal are retained by the authors and/or other copyright owners and it is a condition of accessing publications that users recognise and abide by the legal requirements associated with these rights.

- Users may download and print one copy of any publication from the public portal for the purpose of private study or research.

- You may not further distribute the material or use it for any profit-making activity or commercial gain

- You may freely distribute the URL identifying the publication in the public portal 


\title{
The impact of polar edge terminations of the transition metal dichalcogenide monolayers during vapor growth
}

Qingming Deng ${ }^{\dagger, \ddagger}$, Quoc Huy Thi ${ }^{\ddagger}$, Jiong Zhao ${ }^{\S, \neq, *}$, Seok Joon Yun ${ }^{\ddagger}$, Hyun Kim ${ }^{\ddagger}$, Guibin Chen ${ }^{\dagger}$, Thuc Hue Ly $y^{\mathrm{Il}} \neq, * *$

${ }^{\dagger}$ Physics Department, School of Physics and Electronic Electrical Engineering \& and Jiangsu Key Laboratory for Chemistry of Low-Dimensional Materials, Huaiyin Normal University, Huaian 223300,

Department of Energy Conversion and Storage, Technical University of Denmark, Fysikvej, 2800 Kgs. Lyngby, Denmark.

FIBS Center for Integrated Nanostructure Physics (CINAP), Institute for Basic Science, Sungkyunkwan University, Suwon 440-746, Korea.

${ }^{\S}$ Department of Applied Physics, The Hong Kong Polytechnic University, Kowloon, Hong Kong, China.

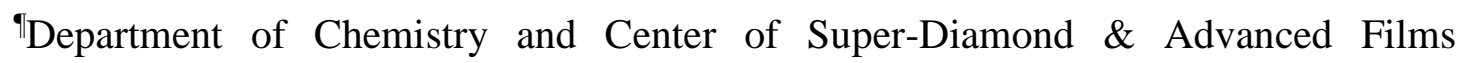
(COSDAF), City University of Hong Kong, Kowloon, Hong Kong, China.

*Correspondence to: jiongzhao@polyu.edu.hk, thuchly@ cityu.edu.hk

\begin{abstract}
The polar edges of two-dimensional monolayer transition metal dichalcogenides (TMD) and their alloys are examined by the combined theoretical (density functional theory) and experimental approaches. For these polar edges, the growth reaction energies between different edge terminations are considered instead of the surface free energies. Due to different energy evolutions during growth on the zigzag edges between $\mathrm{MoS}_{2}$ and $\mathrm{WS}_{2}$, the S-ZZ edges in $\mathrm{WS}_{2}$ monolayer flakes more easily decompose into sawtooth-like edges in $\mathrm{M}-\mathrm{ZZ}$ type as compared to the $\mathrm{MoS}_{2}$ monolayer, thus the
\end{abstract}


hexagonal morphology can be seen more often in $\mathrm{WS}_{2}$. Moreover, the observed anisotropic short range order in the $\mathrm{MoS}_{2} / \mathrm{WS}_{2}$ alloys are originated from the freezed edge configurations during growth, explainable by the growth kinetics and thermodynamics of the Mo-ZZ-edges. The determination of the growing edge terminations is of great importance for the controllable synthesis of the emergent twodimensional TMD materials.

Keywords: Tungsten disulfide, Molybdenum disulfide, growth, polar edge, TEM, DFT.

Knowledge about the exact atomic terminations at the growing front is the key for crystal growth and design. For elementary substance crystals, such as $\mathrm{Si}$ or $\mathrm{Ge}$, to know the compositions of the free surfaces is enabled by the Wulff constructions ${ }^{1,2}$, the morphology of the single crystals can be straightforwardly derived according to the exact surface energies ${ }^{2}$. However, multicomponent compounds involve much more possibilities of surface terminations ${ }^{3}$. Moreover, polar surfaces widely existed in compound semiconductors ${ }^{3,4}$, such as the most representative wurtzite $(0001) /(000-1)$ surfaces. Because of the intrinsic difficulty in the determination of polar surface free energy $^{5}$ as well as the tendency of reconstructions on polar surfaces due to electron counting rule unsatisfied ${ }^{3,4}$, the polar surface terminations are usually obscure.

In the current work we focus on the polar edges (polar surfaces for two dimensional materials) in transition metal dichalcogenides (TMD) monolayers ${ }^{6}$. The emergent two dimensional (2D) materials have many fascinating properties ${ }^{6,7}$, amongst which the mirror symmetry broken (reduction from six-fold into three-fold symmetry) in the single-atomic-layered TMD is very impressive ${ }^{8,9}$. Basically it is the origin of polarizations such as the piezoelectric ${ }^{9}$ and valleytronic ${ }^{8}$ properties in monolayer TMDs. Owning to absence of center inversion symmetry, the TMD monolayer crystals have polar (10-10) edges. It's well known the surfaces are critical for crystal growth. Nowadays TMD monolayers including $\mathrm{MoS}_{2}$ or $\mathrm{WS}_{2}$ can been readily synthesized via the chemical vapor deposition (CVD) methods ${ }^{10-12}$, however, lacking of controllable synthesis protocol especially the morphology, size and defect control for the final products has become the major barrier for future applications ${ }^{13,14}$.

As already reported, the $\mathrm{MS}_{2}(\mathrm{M}=\mathrm{Mo}$ or $\mathrm{W})$ monolayers (Figure 1a) were grown on $\mathrm{Si} / \mathrm{SiO}_{2}$ or sapphire substrates, employing Sulphur and $\mathrm{MO}_{\mathrm{x}}$ as the sources ${ }^{10-12}$. The ex 
situ transmission electron microscopy (TEM) $)^{15,16}$ and scanning tunneling microscopy $(\mathrm{STM})^{17,18}$ on $\mathrm{MoS}_{2}$ monolayers have exhibited that Mo-zigzag (Mo-ZZ) edges are favorable whilst the S-ZZ edges can be observed in the early growth stages or accompanied with dopant cobalt atoms (The definition of the edge types can be found in Figure 1b). As the final shapes of monolayer TMDs are either equatorial triangle or hexagonal which means the other edges have much higher energies than $\mathrm{ZZ}$ edges, we will only consider the $\mathrm{ZZ}$ edges ( $\{10-10\}$ surfaces) here. In the first part of this paper, we would like to address the stability of the possible edge configurations by density functional theory (DFT) calculations ${ }^{19,20}$, and then the edge influences on the experimental morphologies of the $\mathrm{MoS}_{2}$ and $\mathrm{WS}_{2}$ flakes will be compared and rationalized. More interestingly, the particular short range order (SRO) in TMD alloys ${ }^{21}$ can be elaborated based on the stability and growth kinetics between these free edge structures. Our congruent experimental and theoretical approaches successfully revealed the most stable terminations of the free edges in TMD monolayers during vapor growth.

As mentioned above, the $\mathrm{ZZ}$ edges of $\mathrm{MS}_{2}$ can be divided into $\mathrm{M}-\mathrm{ZZ}$ and $\mathrm{S}-\mathrm{ZZ}$ edges (Figure 1c). The vapor phase growth conditions are close to equilibrium with high crystallinity, faceting and less defects, and the possible edge configurations for each edge has three sub-types, they are M-ZZ, M-ZZ-1S, M-ZZ-2S; S-ZZ, S-ZZ-M and SZZ-MS, fully illustrated in the schematics in Figure 1c. The DFT calculations give the relaxed atomic configurations as well as the total energies. Methods and details related to DFT calculation are appended in the experimental and supplementary sections. The reaction (growth) pathways follow as: $\mathrm{M}-\mathrm{ZZ}+\mathrm{S} \rightarrow \mathrm{M}-\mathrm{ZZ}-1 \mathrm{~S}$; M-ZZ-1S+S $\rightarrow \mathrm{M}-\mathrm{ZZ}-2 \mathrm{~S}$; $\mathrm{M}-\mathrm{ZZ}-\mathrm{S}+\mathrm{M} \rightarrow \mathrm{M}-\mathrm{ZZ}$, and $\mathrm{S}-\mathrm{ZZ}+\mathrm{M} \rightarrow \mathrm{S}-\mathrm{ZZ}-\mathrm{M} ; \quad \mathrm{S}-\mathrm{ZZ}-\mathrm{M}+\mathrm{S} \rightarrow \mathrm{S}-\mathrm{ZZ}-\mathrm{MS} ; \quad \mathrm{S}-\mathrm{ZZ}-$ $\mathrm{MS}+\mathrm{S} \rightarrow \mathrm{S}-\mathrm{ZZ}$ (Figure 1d). The reaction energy (enthalpy) for each step is then derived (Figure 1d). In high temperature vapor growth, the Mo and $\mathrm{S}$ atoms are assumed to be freely diffused on the edges and fully supplied which will not restrict the reaction speed.

The polar surface energy cannot be straightforwardly derived by the slab models in DFT calculations ${ }^{22}$ or other alternative methods ${ }^{9}$ which can give big errors. we therefore try to avoid using the absolute surface free energy (the edge energy for $2 \mathrm{D}$ materials) ${ }^{15-}$ ${ }^{18}$, but comparing the edge stability by the reaction energies (details in SI). The reaction energies can be reckoned as the activation energies for the inverse process during growth, the lower the energy release for one step, the more unstable for the reactant and more stable for the product. Based on the reaction energy for each step, the M-ZZ-1S edges are the most stable for both $\mathrm{MoS}_{2}$ and $\mathrm{WS}_{2}$, as the M-ZZ-1S+S $\rightarrow \mathrm{M}-\mathrm{ZZ}-2 \mathrm{~S}$ reactions have the lowest energy change (Figure 1d) among all the reactions on both $\mathrm{M}-\mathrm{ZZ}$ and S-ZZ edges, which means this is the rate determining step (RDS). Moreover, 
it is also noted the growth of M-ZZ edge is more difficult while the growth of S-ZZ edge is easier for $\mathrm{WS}_{2}$ than $\mathrm{MoS}_{2}$, as the $\mathrm{S}-\mathrm{ZZ}-\mathrm{MS}+\mathrm{S} \rightarrow \mathrm{S}-\mathrm{ZZ}$ reaction (RDS for growth on $\mathrm{S}-\mathrm{ZZ}$ edge) release larger energy $(4.33 \mathrm{eV})$ for $\mathrm{WS}_{2}$ than for $\mathrm{MoS}_{2}(3.98 \mathrm{eV})$, and the RDS on M-ZZ edge release smaller energy for $\mathrm{WS}_{2}(3.21 \mathrm{eV})$ than $\mathrm{MoS}_{2}(3.43 \mathrm{eV})$ (Figure 1d). Therefore, the M-ZZ-1S termination will dominate in growth by the DFT calculation, and the relative unstable S-ZZ edges have higher possibility to decompose into sawtooth-like M-ZZ edges (Figure 1e) in $\mathrm{WS}_{2}$ than in $\mathrm{MoS}_{2}$ due to M-ZZ-S type edge is more stable (grow more slowly) for $\mathrm{WS}_{2}$ than $\mathrm{MoS}_{2}$.

For more sophisticated reactants, we have also considered the $\mathrm{M}$ or $\mathrm{S}$ dimer atomic species added on the edges during growth. By DFT calculations, the dissociation energies of the Mo, $\mathrm{W}$, and $\mathrm{S}$ dimers are $6.24 \mathrm{eV}, 6.13 \mathrm{eV}, 5.26 \mathrm{eV}$, respectively. Therefore if considering these dimers as the reactants, the reaction energy differences between $\mathrm{MoS}_{2}$ and $\mathrm{WS}_{2}$ edges will just be slightly changed, by around 6.24$6.13) / 2=0.05 \mathrm{eV} /$ atom, the relative stabilities of the edges are not affected.

The morphology of TMD monolayer is sensitive with the growth temperature, vapor pressure, substrate and growth time ${ }^{10-13}$. The microscopic characterizations on the postgrowth samples ${ }^{15-18}$ may not provide the exact edge configurations during growth because the ambient environment is different from the vapor pressure conditions inside the growth chamber. However, the edge terminations during growth can determine the shape and morphology of the flakes in the final product, which cannot be changed by ambient conditions. We therefore carry out the morphology analysis of the experimental $\mathrm{MoS}_{2}$ and $\mathrm{WS}_{2}$ flakes, for testing the stability results acquired by DFT calculations above.

Micrographs of our vapor phase grown $\mathrm{MoS}_{2}$ and $\mathrm{WS}_{2}$ monolayer flakes are presented in Figure 2. The growth methods of $\mathrm{MoS}_{2}$ and $\mathrm{WS}_{2}$ follows our previous publications ${ }^{11,23}$. In general, the $\mathrm{MoS}_{2}$ is easier to form triangles (Figure 2a,b) in most of the conditions, in contrast $\mathrm{WS}_{2}$ can grow hexagons (Figure 2c,d) more frequently. Figure $2 \mathrm{~b}$ show the $\mathrm{MoS}_{2}$ straight edge which have been determined as Mo-ZZ edge by TEM (Supplementary Figure S2). Through TEM analysis on $\mathrm{WS}_{2}$ hexagons, we confirmed the W-ZZ edge is straight (Figure 2e), while the S-ZZ edge is mostly sawtooth-like (Figure $2 \mathrm{f}, \mathrm{g})^{23}$. It is reasonable that the $\mathrm{WS}_{2}$ is easier to have decomposed sawtooth S-ZZ edges because the growth speed difference between the S-ZZ edge and $\mathrm{W}-\mathrm{ZZ}$ edge is larger in $\mathrm{WS}_{2}$ than in $\mathrm{MoS}_{2}$ by our DFT results (Figure 1d). Owning to the decomposition of $\mathrm{S}-\mathrm{ZZ}$ edge of $\mathrm{WS}_{2}$, the $\mathrm{WS}_{2}$ flakes become hexagonal. Hence, the shapes and edge morphology of the experimental $\mathrm{MoS}_{2}$ and $\mathrm{WS}_{2}$ flakes are in agreement with the most stable edge terminations (M-ZZ-1S) by DFT. 
For larger sized flakes, the triangle shape will naturally be less favored than the hexagonal shape as the increasing barrier induced by mass supply. Actually round/circle shape is most favorable with smallest barrier from kinetic point of view. The growth of smooth edges require a close-to-equilibrium growth conditions, and a condition with less kinetic barriers, however, the corner and center part of a long smooth edge in triangle flake for growth will bring in different reactant consumption rates (to keep the smooth and straight edge) and local reactant concentration inhomogeneity, leading to higher kinetic barriers. The trend of hexagonal shape induced by kinetics is also one condition for S-ZZ edge decomposition into sawtooth W-ZZ edges, otherwise only triangle shapes can occur if no kinetics are involved. A more comprehensive theoretical simulation including all kinetic factors and all possible reactants will be published later.

Moreover, the edge terminations during growth can be correlated with the superstructures in 2D TMD alloys. The short range order (SRO) in microstructure of binary disordered alloy (substitutional solid solution) is common and critical for a number of engineering purposes ${ }^{24,25}$. $\mathrm{MoS}_{2}$ and $\mathrm{WS}_{2}$, which have exactly the same lattice constant (3.18 $\AA$ by DFT) in monolayer (less than $1.3 \%$ difference), unsurprisingly lead to epitaxial coherent interface at the $\mathrm{MoS}_{2}-\mathrm{WS}_{2}$ heterojunction ${ }^{26}$ or formation of infinite disordered solid solutions of $\mathrm{Mo}_{\mathrm{x}} \mathrm{W}_{1-\mathrm{x}} \mathrm{S}_{2}$ at any compositions $(0<x<1)^{27}$. Similarly, mono-atomic layer graphene-BN composite $\mathrm{B}_{1-\mathrm{x}} \mathrm{C}_{\mathrm{x}} \mathrm{N}_{1-\mathrm{x}}$ is another example of binary system in $2 \mathrm{D}$ materials ${ }^{28}$, but the miscible ability of $\mathrm{B}_{1-\mathrm{x}} \mathrm{C}_{\mathrm{x}} \mathrm{N}_{1-\mathrm{x}}$ is much lower than $\mathrm{Mo}_{\mathrm{x}} \mathrm{W}_{1-\mathrm{x}} \mathrm{S}_{2}$, with easy phase separations. One previous report has discussed about in mono-atomic layer $\mathrm{Mo}_{\mathrm{x}} \mathrm{W}_{1-\mathrm{x}} \mathrm{S}_{2}$ the first nearest neighbor of the metal atom (Mo or $\mathrm{W}$ ) sub-lattice is randomly mixed ${ }^{29}$. While another work has mentioned about the anisotropic stripe structures in $\mathrm{Mo}_{\mathrm{x}} \mathrm{W}_{1-\mathrm{x}} \mathrm{S}_{2}$ induced by the phase separation at the growth edges ${ }^{30}$.

Our binary alloy $\mathrm{Mo}_{\mathrm{x}} \mathrm{W}_{1-\mathrm{x}} \mathrm{S}_{2}$ sample is also grown by $\mathrm{CVD}$ method on $\mathrm{Si} / \mathrm{SiO}_{2}$ substrate at high temperatures with mixed sources of tunable compositions, the fabrication specifications are similar to our previous methods for TMDs ${ }^{11}$. Typical high-mag TEM images for $\mathrm{Mo}_{\mathrm{x}} \mathrm{W}_{1-\mathrm{x}} \mathrm{S}_{2}$ are presented in Figure 3a. Each Mo and $\mathrm{W}$ atom (for sulphur, two sulphur atoms in the same column) can be unambiguously determined and their positions are digitalized by the high angle annular dark field (HAADF) images (Figure $3 b$ ). The contrast of atoms in HAADF is monotonically dependent on the atomic numbers $(\mathrm{Z})^{31}$. SRO in alloy is usually described by the Warren-Cowley parameters $\left(\alpha_{l m n}\right)^{32}$,

$$
\alpha_{l m n}=1-\frac{P_{l m n}^{A B}}{c^{B}}
$$


where $P_{l m n}^{A B}$ is the conditional probability of atom B appear in the neighboring shell lmn of atom $\mathrm{A}$ in $\mathrm{AB}$ alloy and $c^{B}$ is the atomic fraction. $\alpha_{l m n}$ approaches zero if two atoms separated by vector [lmn] have no pair correlations, and negative/positive $\alpha_{l m n}$ means the specific configurations between $\mathrm{A}$ and $\mathrm{B}$ atoms are favored/unfavored over the random distribution. The measurement details of all the $\alpha_{l m n}$ are appended in Methods section. Figure 3c presents the results of $\alpha$ for $l m n$ from first nearest neighbor to seven times lattice parameter distance, accompanied by the schematic for each $\alpha$. We can see for both W-W in $\mathrm{Mo}_{0.44} \mathrm{~W}_{0.56} \mathrm{~S}_{2}$ case, $\alpha(1 / 3,1 / 3,-2 / 3), \alpha(2 / 3,2 / 3,-4 / 3), \alpha(1,1,-2)$, $\alpha(4 / 3,4 / 3,-8 / 3)$ are all significantly less than zero while the others are close to zero, which means the same kind of atoms are preferentially located along the zigzag direction [11-2], leading to straight chains of $\mathrm{W}$ atoms. There is similar trend for Mo atoms. The summary for all the other $\alpha$ for different compositions of alloy are appended in Table $S 1$ in the supplementary materials. We found the compositions near $x=0.5$ has the most pronounced SRO, and SRO widely exist for most of the alloys.

Further, radar plot can show the radial direction dependence of $\alpha$ (Figure 4). For most of the $\alpha$, there is one "principle axis" in which the SRO is much more prominent than the others. This principle axis is always correlated with the growing direction or the free edge directions of sample in the region of interest (see Figure 4a,b). The growth direction of the free edges of the examined area by HAADF imaging is determined by its location of the whole triangle samples by low magnification TEM images (Figure $4 b$ ). Therefore, our obtained full set of $\alpha$ of $\mathrm{Mo}_{\mathrm{x}} \mathrm{W}_{1-\mathrm{x}} \mathrm{S}_{2}$ (Figure $4 \mathrm{c}$ ) implies the tendency to form atomic $\mathrm{W}$ chains or Mo chains perpendicular to the growth direction. The fast Fourier transform (FFT) of the high resolution images (Supplementary Figure S3) also indicate the existence of chains by the elongated central streaks along growth directions.

Meanwhile, we also checked the SRO in the alloy of $\mathrm{VSe}_{2} / \mathrm{WSe}_{2}\left(\mathrm{~W}_{0.75} \mathrm{~V}_{0.25} \mathrm{Se}_{2}\right)$ (Figure 5a), made by alloying of $\mathrm{VSe}_{2}$ and $\mathrm{WSe}_{2}$ (see methods). This sample exhibit neither anisotropy nor SRO (Figure 5b), and the morphology of the flakes are irregular and inapplicable to determine the edges or facets during growth, implying the faceting and edge effects like in $\mathrm{Mo}_{\mathrm{x}} \mathrm{W}_{1-\mathrm{x}} \mathrm{S}_{2}$ are essential in the vapor growth for alloys. In terms of $\mathrm{Mo}_{\mathrm{x}} \mathrm{W}_{1-\mathrm{x}} \mathrm{S}_{2}$, the layer-by-layer growth mode which causes atomically flat edges on the as-synthesized samples can "freeze" the W/Mo atom positions after they have been grown, without aging effect. In addition, W/Mo atomic layer are sandwiched by two Sulphur layers, so the diffusion or interchange of W/Mo atoms require rearrangement of surrounding Sulphur atoms, requiring high energy supply and unlikely to occur. Therefore, the superstructures such as the SRO are actually determined by the edge terminations during growth. 
We performed series of DFT calculations on the $\mathrm{Mo}_{\mathrm{x}} \mathrm{W}_{1-\mathrm{x}} \mathrm{S}_{2}$ edge terminations $(\mathrm{x}=$ 0.5) in supplementary Figure S4-S5. The results show these edges have significant perpendicular relaxations. For M-ZZ edges, the second row of W-S bond length of Mo$\mathrm{ZZ}$ perpendicular to the edge is $2.5 \%$ elongated, while for Mo-S bond of W-ZZ this elongation is $3 \%$ by comparison to the bulk value (Figure S4). The bond lengths of bare metals at the first row and S are 2.372 and $2.373 \AA$ which are 0.031 and $0.035 \AA$ shorter than the corresponding bulk phase for Mo and $\mathrm{W}$ in average respectively. We note that the energies of Mo-ZZ are lower than W-ZZ $(0.93 \mathrm{eV})$ by introducing the same composition of alloys, and the energies of phase mixed edges are in range of Mo-ZZ and W-ZZ edges (Fig.S4). The energy difference by evaluating the total energies of Mo-ZZ and W-ZZ minus 2 times energy of MoW-ZZ edges is quite small $(+0.03 \mathrm{eV} / \mathrm{atom})$ at the edges indicating the coexistence of these three phases. Besides, various distributions at the edges also reveal separated and mixed phases are isoenergetic represented in Fig.S5. Doping Co atoms in $\mathrm{MoS}_{2}$ ribbon at the edge significantly decreases the stabilities by $2.14 \mathrm{eV} /$ atom. Interestingly, in such Co-Mo alloys the pairing configuration $\mathrm{Co}-\mathrm{Co}-\mathrm{Mo}-\mathrm{Mo}$ is more energetically favorable by $0.03 \mathrm{eV} /$ atom than the alternate configuration $\mathrm{Co}-\mathrm{Mo}-\mathrm{Co}-\mathrm{Mo}$ as shown as in Fig. S6. Thus, from the kinetic point of view, one explanation of our observed SRO is that the predominant competing Mo-ZZ edges form at the beginning. With the ratio of $\mathrm{W}$ in local growth atmosphere becoming higher, W-ZZ edges become preferable. Previous study $^{33}$ suggests that complete M-ZZ edges may only occur under highly reductive conditions or at high temperature, so the formation mechanism is still an open question as edge states not only relates to the thermostability, but also influenced by the different conditions, such as the ratio of mixed metals, pressure for each sources, flow direction/rate, temperatures, etc.

In summary, upon analysis on the CVD grown TMD materials, including monolayer $\mathrm{MoS}_{2}, \mathrm{WS}_{2}$ as well as the 2D alloys, we identified the different shapes and edges between $\mathrm{MoS}_{2}$ and $\mathrm{WS}_{2}$ flakes and the pronounced anisotropic SRO in their disordered alloys. By employing the dedicated DFT calculations and reaction energies between different edge terminations, the results converged with the experimental findings that M-ZZ-1S is the main edge termination for the $\mathrm{MoS}_{2}$ and $\mathrm{WS}_{2}$ monolayers during vapor growth. In terms of methodology, we have proposed a new approach to validate the theoretically acquired polar edge terminations during growth. The post-growth morphologies and superstructures which can be easily utilized for this purpose. The understanding of the edge structure will give a better chance for the growth control of these novel 2D materials. In addition, the noteworthy edge-freezed long range SRO (until $2 \mathrm{~nm}$ ) not only affect the physical properties of $\mathrm{Mo}_{\mathrm{x}} \mathrm{W}_{1-\mathrm{x}} \mathrm{S}_{2}$ (ref.30), or similar compounds like $\mathrm{W}_{\mathrm{x}} \mathrm{V}_{1-\mathrm{x}} \mathrm{Se}_{2}, \mathrm{MoS}_{\mathrm{x}} \mathrm{Se}_{2-\mathrm{x}}$ (ref. 34), $\mathrm{Mo}_{\mathrm{x}} \mathrm{W}_{1-\mathrm{x}} \mathrm{Se}_{2}$ (ref. 35), $\mathrm{Mo}_{\mathrm{x}} \mathrm{W}_{1-\mathrm{x}} \mathrm{Se}_{\mathrm{y}} \mathrm{S}_{2-\mathrm{y}}$ 
(ref. 36), etc., but also give some new insights: in-operando engineering specifically on the surface/edge during growth might be a new route to modulate structures for random alloys, which is inaccessible by global heat treatment methods previously.

\section{Experimental Section:}

Density functional theory (DFT) calculations. The spin-polarized density function theory calculations were carried out by the Vienna ab initio simulation package (VASP) program package ${ }^{37}$, ${ }^{38}$ with the projector augmented wave (PAW) ${ }^{20,39}$ to explore the growth mechanisms of $\mathrm{Mo}_{\mathrm{x}} \mathrm{W}_{1-\mathrm{x}} \mathrm{S}_{2}$ monolayer. The exchange-correlation functions were described with the generalized gradient approximation (GGA) in the form of the Perdew, Burke, and Ernzernhof (PBE) functional ${ }^{19}$. The kinetic energy cutoff for the plane-wave basis set was chosen as $500 \mathrm{eV}$. The interlayer distance was set to be more than $15 \AA$, which is large enough to minimize the artificial interlayer interactions. The electronic SCF tolerance is set $10^{-5} \mathrm{eV}$. Fully relaxed geometries and lattice constant were obtained by optimizing all atomic positions until Hellmann-Feynman force was less than $0.02 \mathrm{eV} / \AA$. The $\mathrm{k}$ points samplings were $6 \times 1 \times 1$ in the Brillouin zone for structural optimizations. A dipole correction was added to avoid interactions between periodic images of the slab along the $\mathrm{z}$ direction.

Synthesis of TMD monolayer alloys. For synthesizing monolayer $\mathrm{W}_{1-\mathrm{x}} \mathrm{V}_{\mathrm{x}} \mathrm{Se}_{2}$ alloy, we introduce two-zone furnace atmospheric CVD system for controlling temperature of each zone separately. 0.05 gram of vanadium oxide (Sigma, 223794, $\mathrm{V}_{2} \mathrm{O}_{5}$ ) and 0.1 gram of tungsten trioxide (Sigma, 204781, $\mathrm{WO}_{3}$ ) powers were loaded to ceramic crucible then $\mathrm{SiO}_{2} / \mathrm{Si}$ wafer is placed to face each other in substrate zone. 0.3 gram of selenium (Sigma, 229865, Se) was loaded in selenium zone. The Substrate zone are heated first then maintained at $500{ }^{\circ} \mathrm{C}$ for 10 min to remove any water or oxygen absorption on metal power precursors. After that, the temperature of substrate and selenium zone are elevated upto $750{ }^{\circ} \mathrm{C}$ and $400{ }^{\circ} \mathrm{C}$ and maintained for 10 minutes. When temperature of both zone are reached to its maximum temperature, 10 sccm of hydrogen gas were injected to chamber because vanadium and tungsten oxide are not reduced only by selenium but reduced to $\mathrm{MSe}_{2}$ with presence of hydrogen gas. The temperature of both zone are naturally cooled down to room temperature by opening the furnaces. $\mathrm{W}_{0.44} \mathrm{Mo}_{0.56} \mathrm{~S}_{2}$ was grown on $\mathrm{SiO}_{2} / \mathrm{Si}$ wafer by atmospheric $\mathrm{CVD}$ process using the same method as synthesis of alloying vanadium and tungsten diselenide.

Scanning electron microscopy (SEM). Field-emission scanning electron microscopy (FESEM) (JSM7000F, Jeol, Japan) was used to examine the surface morphology of samples at different accelerating voltages to obtain a high level of contrast at different magnifications. An accelerating voltage of $10 \mathrm{keV}$ was used to obtain sufficiently pronounced signals while retaining sensitivity to the sample surface. 
TEM sample preparation. The CVD tungsten disulphide was transferred on a hole with 1.2 $\mu \mathrm{m}$ in diameter in $\mathrm{Cu}$ quantifoil TEM grid (Product No. 658-200-CU) by PMMA-assistant method. Thin layer PMMA was spin-coated on as-grown $\mathrm{MX}_{2}\left(\mathrm{M}: \mathrm{Mo}, \mathrm{W}, \mathrm{Mo}_{\mathrm{x}} \mathrm{W}_{1-\mathrm{x}}, \mathrm{V}_{\mathrm{x}} \mathrm{W}_{1-}\right.$ x) and $\mathrm{X}: \mathrm{S}$ or $\mathrm{Se}) / \mathrm{SiO}_{2} / \mathrm{Si}$ substrate (2000 rpm, 1min). The $\mathrm{MX}_{2}$ and PMMA support were then detached from the $\mathrm{SiO}_{2} / \mathrm{Si}$ substrate by floating the PMMA/MX $/ \mathrm{SiO}_{2} / \mathrm{Si}$, with the PMMA side up, in a $1 \mathrm{M}$ HF solution. Next, PMMA/MX $\mathrm{MX}_{2}$ was washed by deionized water. The PMMA/MX 2 layer is cooped out in pieces onto TEM grid, then PMMA was removed gently by evaporated acetone (acetone was heated up to $130{ }^{\circ} \mathrm{C}$ ), leaving $\mathrm{MX}_{2}$ suspended freely on holes in TEM grid substrate. Finally, sample was annealing at $180^{\circ} \mathrm{C}$ in a high vacuum $\left(10^{-6}\right.$ Torr) during 12 hours to further remove PMMA.

TEM measurements. TEM experiments were carried out using JEM ARM 200F under $80 \mathrm{kV}$. The acquisition time for dark field (DF) imaging was $1 \mathrm{~s}$ using the smallest objective lens aperture. And the reflex (10-10) was always selected for DF imaging. The HR-TEM imaging acquisition time was $1 \mathrm{~s}$. Annular dark field (ADF)-STEM imaging was conducted with a CEOS aberration-corrector on the same TEM. High-angle annular dark field (HAADF) images were acquired at a $20 \mathrm{mrad}$ convergence angle.

Short range order (SRO) analysis. For $\mathrm{Mo}_{\mathrm{x}} \mathrm{W}_{1-\mathrm{x}} \mathrm{S}_{2}$, we neglected the sublattice of sulphur, and employed the first three indexes in the standard hexagonal four index nomenclature as $\operatorname{lmn}$. By using the high resolution HAADF images, we developed in-house code and digitalized all the atomic positions of Mo and $\mathrm{W}$, then automatically obtained all the $\alpha_{l m n}(\mathrm{Mo}-\mathrm{Mo})$ and $\alpha_{l m n}(\mathrm{~W}-$ W). $\alpha_{l m n}(\mathrm{Mo}-\mathrm{W})$ can be accordingly determined by the relationship $\alpha_{l m n}(\mathrm{Mo}-\mathrm{Mo})+\alpha_{l m n}(\mathrm{Mo}-$ $\mathrm{W})=2$. We intentionally separated the $\alpha_{l m n}$ in different crystal directions in the same $l m n$ shell in order to analyze the directional distributions. The different alloy compositions ranges from $x=0.08$ to $x=0.82$ are considered. Each $\alpha$ is statistically evaluated by more than $10^{5}$ sampling atoms taken by HAADF images.

\section{ASSOCIATED CONTENT}

Supporting Information. Supporting information is available free of charge at http://pubs.acs.org.

\section{AUTHOR INFORMATION}

\section{Corresponding Author}

*E-mail address: (J. Zhao) zhaojiong@gmail.com, (T. H. Ly) thuchue@ skku.edu 


\section{ACKNOWLEDGMENT}

This work was supported by the the National Science Foundation of China (21703076),

the People Programme (Marie Curie Actions) of the European Union's Seventh Framework Programme (FP7/2007-2013) under REA grant agreement no. 609405 (COFUNDPostdocDTU), Natural Science Foundation of Jiangsu Province of China (BK20170466), the Hong Kong Polytechnic University Grant (No. 1-ZE8C), Grant by City University of Hong Kong (9610387, 7200551), and Institute for Basic Science (IBS-R011-D1).

\section{REFERENCES}

(1) Burton, W. K.; Cabrera, N.; Frank, F. C. Philosophical Transactions of the Royal Society A 1951, 243, 299.

(2) Wulff, G. Zeitschrift für Krystallographie und Mineralogie 1901, 34, 449-530.

(3) Kangawa, Y.; Akiyama, T.; Ito, T. et al. Materials 2013, 6, 3309-3360.

(4) Wang, Z.; Pan, N. et al. The Journal of chemical physics 2013, 139, 124704.

(5) Zhang, J.; Zhang, Y.; Tse, K. et al. arXiv 2015, arXiv:1510.08961

(6) Radisavljevic, B.; Radenovic, A.; Brivio, J. et al. Nat. Nanotech. 2011, 6, 147-150.

(7) Mak, K. F.; Lee, C.; Hone, J. et al. Phys. Rev. Lett. 2010, 105, 136805.

(8) Xiao, D.; Liu, G. B.; Feng, W. et al. Phys. Rev. Lett. 2012, 108, 196802.

(9) Wu, W.; Wang, L.; Li, Y. et al. Nature 2014, 514, 470-474.

(10) Li, B.; Gong, Y.; Hu, Z. et al. Angewandte Chemie 2016, 128, 10814-10819.

(11) Han, G. H.; Kybert, N. J.; Naylor, C. H. et al. Nat. Commun. 2015, 6, 6128.

(12) Yun, S. J.; Chae, S. H.; Kim, H. et al. ACS Nano 2015, 9, 5510-5519.

(13) Govind Rajan, A.; Warner, J. H.; Blankschtein, D. et al. ACS Nano 2016, 10, 43304344. 
(14) Artyukhov, V. I.; Hu, Z.; Zhang, Z. et al. Nano Lett. 2016, 16, 3962-3702.

(15) Zhu, Y.; Ramasse, Q. M.; Brorson, M. et al. Angewandte Chemie International Edition 2014, 53, 10723-10727.

(16) Zhu, D.; Shu, H.; Jiang, F. et al. 2D Materials and Applications 2017, 1, 8.

(17) Vang, R. T.; Lauritsen, J. V.; Laegsgaard, E. et al. Chem. Soc. Rev. 2008, 37, 21912203.

(18) Bollinger, M. V.; Lauritsen, J. V.; Jacobsen, K. W. et al. Phys. Rev. Lett. 2001, 87, 196803.

(19) Kresse, G.; Joubert, D. Phys. Rev. B 1999, 59, 1785-1775.

(20) Perdew, J. P.; Burke, K.; Ernzerhof, M. Phys. Rev. Lett. 1996, 77, 3865-3868.

(21) Komsa, H. P.; Krasheninnikov, A.V. J. Phys. Chem. Lett. 2012, 3, 3652-3656.

(22) Skriver, H. L.; Rosengaard, N. M. Phys. Rev. B 1992, 46, 7157.

(23) Ly, T. H.; Yun, S. J.; Thi, Q. H.; Zhao J. ACS NANO 2017, 11, 7534.

(24) Falicov, L. M.; Yndurain, F. Phys. Rev. B 1975, 12, 5664.

(25) Schönfeld, B. Progress in materials science 1999, 44, 435-543.

(26) Gong, Y.; Lin, J.; Wang, X. et al. Nat. Mater. 2014, 13, 1135-1142.

(27) Chen, Y.; Xi, J., Dumcenco, D. O. et al. ACS Nano 2013, 7, 4610-4616.

(28) Lu, J.; Zhang, K.; Liu, X. F. et al. Nat. Comm. 2013, 4, 2681.

(29) Dumcenco, D. O.; Kobayashi, H.; Liu, Z. et al. Nat. Comm. 2013, 4, 1351.

(30) Azizi, A.; Wang, Y.; Lin, Z. et al. Nano Lett. 2016, 16, 6982-6987.

(31) Pennycook, S. J. Ultramicroscopy 1989, 30, 58-69.

(32) Norman, N.; Warren, B. E. J. Appl. Phys. 1951, 22, 483-486.

(33) Prodhomme, P.-Y.; Raybaud P.; Toulhoat, H. Journal of Catalysis, 2011, 280, 178-195.

(34) Mann, J.; Ma, Q.; Odenthal, P. M. et al. Adv. Mater. 2014, 26, 1399-1404.

(35) Tongay, S.; Narang, D. S.; Kang, J. et al. Appl. Phys. Lett. 2014, 104, 012101. 
(36) Li, M. Y.; Shi, Y.; Cheng, C. C. et al. Science 2015, 349, 524-528.

(37) Kresse, G.; Hafner, J. Phys. Rev. B 1993, 47, 558-561.

(38) Kresse, G.; Furthmüller, J. Phys. Rev. B 1996, 54, 11169-11186.

(39) Blöchl, P. E. Phys. Rev. B 1994, 50, 17953-17979.

\section{FIGURE LEGENDS:}

Figure 1 | The theoretical results for $\mathrm{MS}_{2}(\mathrm{M}=\mathrm{W}$ or Mo) edge terminations. a, The DFT calculated unit cells for $\mathrm{MoS}_{2}$ and $\mathrm{WS}_{2}$ monolayers. b, Scheme of the two type of zigzag edges (M-ZZ and S-ZZ) in one quadrilateral flake. c, The DFT calculated possible edge terminations on ZZ edges. d, The DFT calculated growth reaction energies of different steps during growth, for both M-ZZ and S-ZZ. e, Scheme of the decomposed sawtooth S-ZZ edge, using M-ZZ termination as example. Mo, W, and $\mathrm{S}$ atoms are colored in purple, cyan, and yellow, respectively.

Figure 2 | The experimental monolayer $\mathbf{M S}_{2}$ morphologies. a, The triangle-shape $\mathrm{MoS}_{2}$ monolayer flakes, scale bar is $20 \mu \mathrm{m}$. b. The TEM dark field image showing the straight edge of $\mathrm{MoS}_{2}$ flake in a, scale bar is $100 \mathrm{~nm}$. c, The hexagonal-shape $\mathrm{WS}_{2}$ monolayer flake, sacle bar is $20 \mu \mathrm{m}$. d, TEM bright field image showing the faceted edges (W-ZZ and S-ZZ determined by diffraction method or high resolution STEM method), scale bar is $10 \mu \mathrm{m}$. e, The TEM dark field image for straight $\mathrm{W}-\mathrm{ZZ}$ edge in $\mathrm{WS}_{2}$ monolayer in $\mathbf{d}$, scale bar is $200 \mathrm{~nm}$. f, $\mathbf{g}$, The TEM dark field images for sawtoothshape $\mathrm{S}-\mathrm{ZZ}$ edge in $\mathbf{d}$, scale bar is $200 \mathrm{~nm}$.

Figure 3 | The short range order (SRO) analysis in $\mathrm{WS}_{2} / \mathrm{MoS}_{2}$ alloy, a, high resolution annular dark field (ADF) image for the $\mathrm{W}_{0.44} \mathrm{Mo}_{0.56} \mathrm{~S}_{2}$ monolayer sample, scale bar is $1 \mathrm{~nm}$. b. Digitalized $\mathrm{W}$ atomic positions corresponding to a. c, Calculated Cowley-Warren SRO coefficients for $\mathrm{W}$ in the $\mathrm{W}_{0.44} \mathrm{Mo}_{0.56} \mathrm{~S}_{2}$ sample. Each direction is illustrated by atomic model close to the corresponding data point. And lower right insets show the magnified ADF image and the atomic models.

Figure 4 | The anisotropic SRO in $\mathbf{M o}_{\mathbf{x}} \mathbf{W}_{1-x} S_{2}$ flake. a, Scheme for the growth and facets of triangle alloy flake, and the analyzed region of interest can be associated with the growth direction by the shape and edge. $\mathbf{b}$, The TEM image for the triangle $\mathrm{Mo}_{\mathrm{x}} \mathrm{W}_{1-}$ ${ }_{\mathrm{x}} \mathrm{S}_{2}$ flake, scale bar is $2 \mu \mathrm{m}$. c, The radar plot showing the Cowley-Warren coefficients in space of the sample $\mathrm{W}_{0.44} \mathrm{Mo}_{0.56} \mathrm{~S}_{2}$. The preferred directions are highlighted in red 
while other directions in blue. The edge and growth direction for this sample is marked by the arrows.

Figure 5 | The experimental results for $W_{x} V_{1-x} S_{2}$ alloy. a, High resolution ADF image for the $\mathrm{W}_{\mathrm{x}} \mathrm{V}_{1-\mathrm{x}} \mathrm{Se}_{2}$, scale bar is $1 \mathrm{~nm}$. $\mathbf{b}$, The Cowley-Warren coefficients obtained from the sample $\mathrm{W}_{0.75} \mathrm{~V}_{0.25} \mathrm{Se}_{2}$, the lower left inset shows the optical micrograph for the flake, scale bar is $50 \mu \mathrm{m}$, the lower right inset shows the magnified atomic-scale ADF image. 


\section{Figures:}

\section{Figure 1}

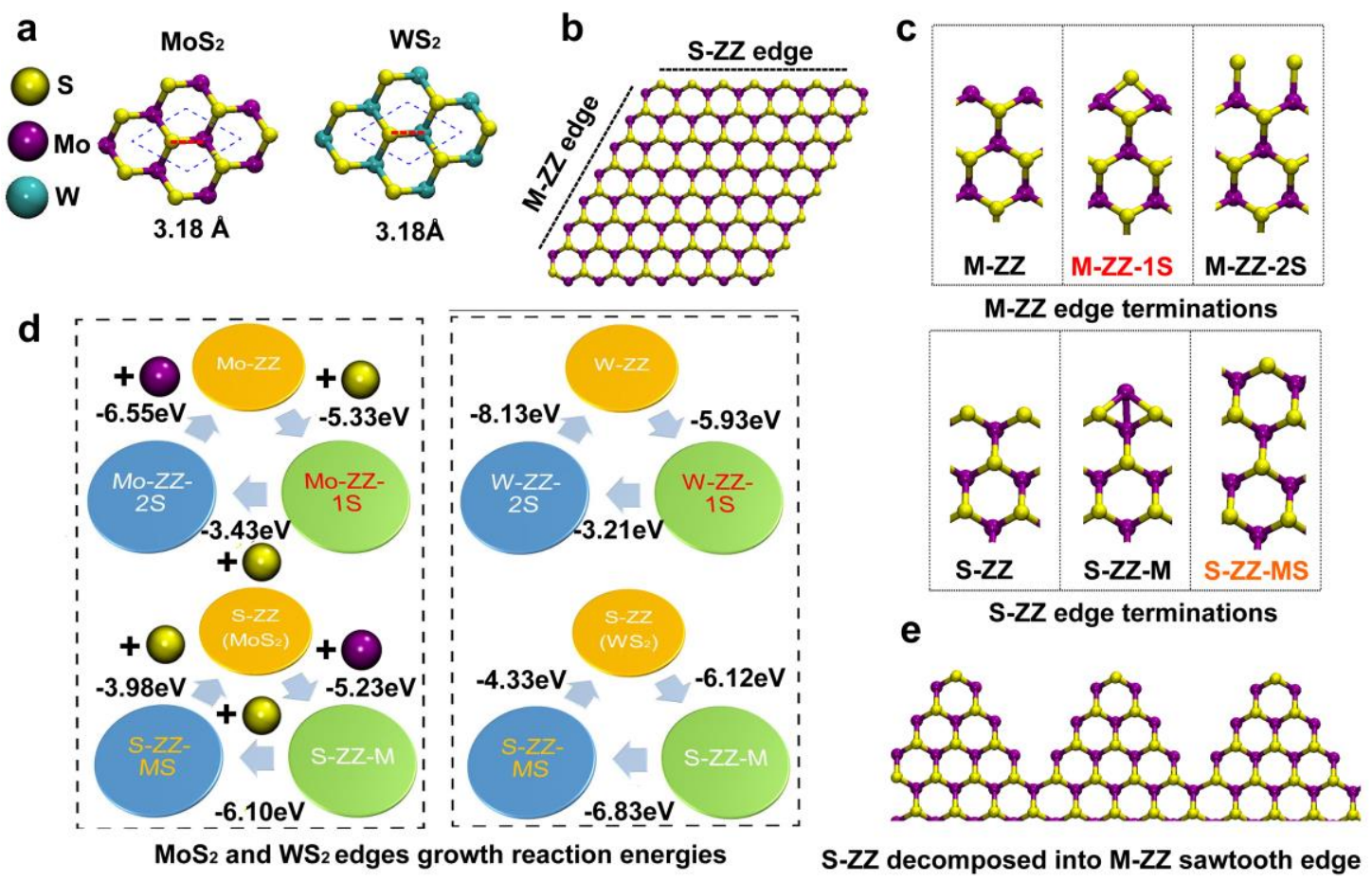

Figure 2

a

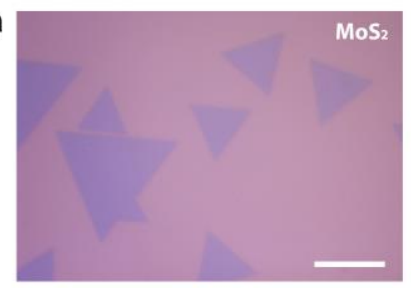

b

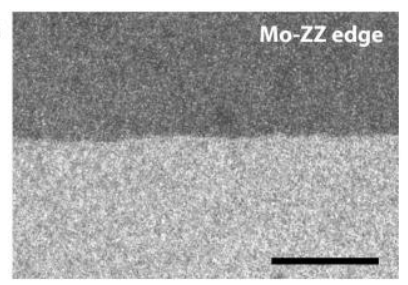

c

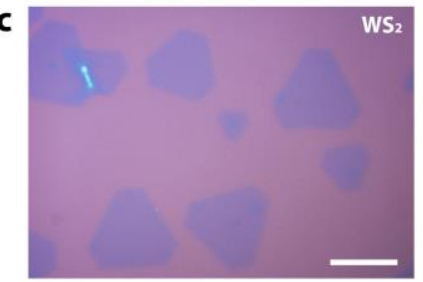

d

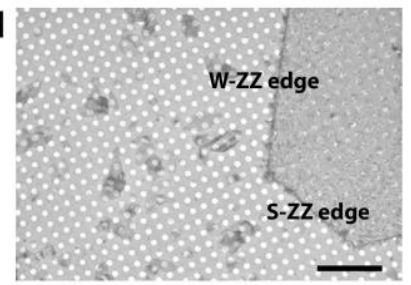

e

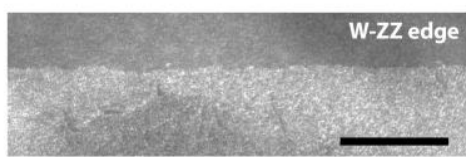

f

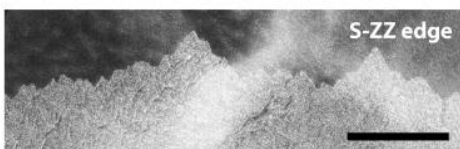

g

S-ZZ edge

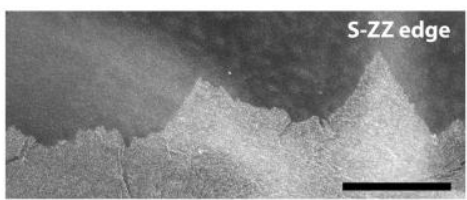


Figure 3
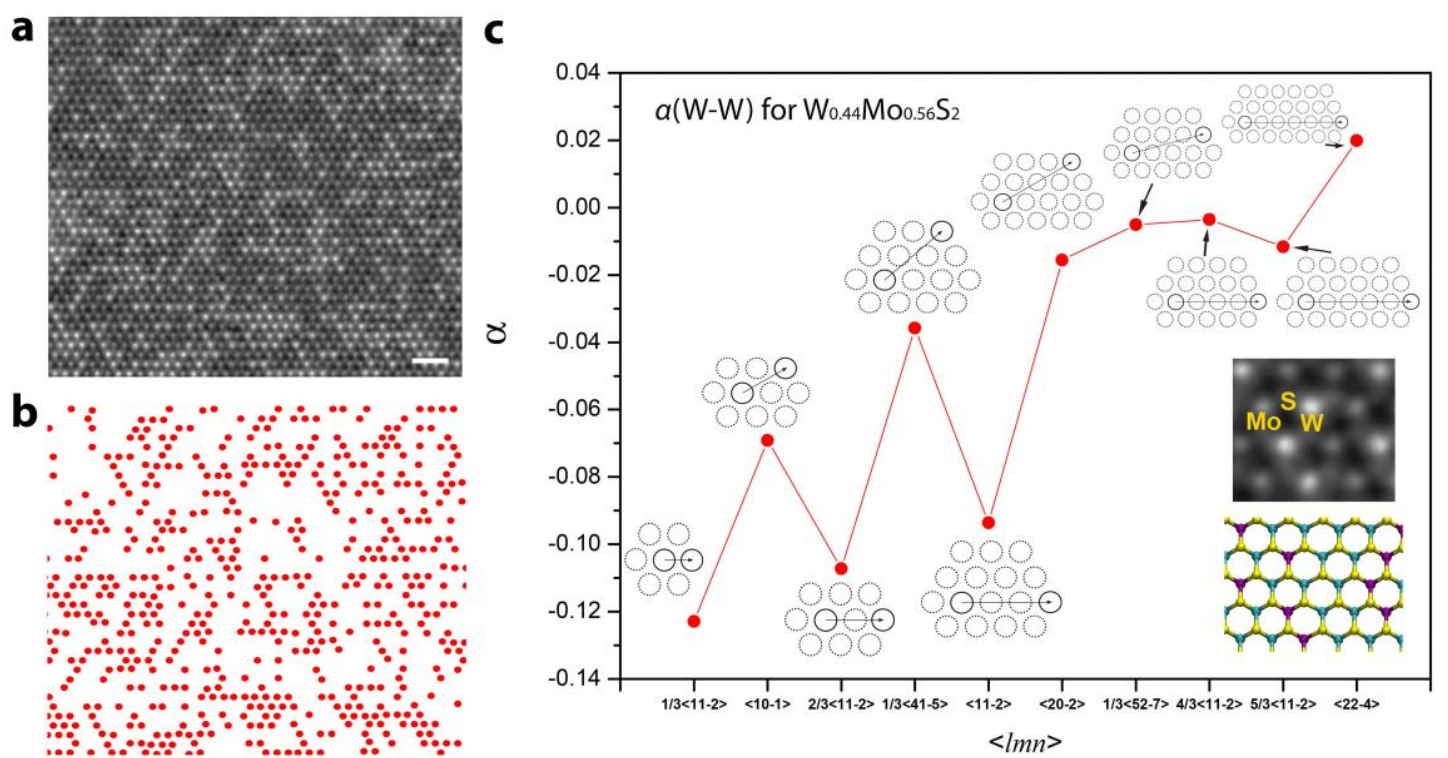

b. $\because \because \therefore \because \therefore \because \because \because \because \cdots$

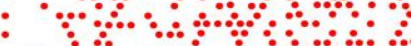
$\therefore \because \cdots \cdots$ $\because \because \because \quad \because$ $\therefore \cdots$

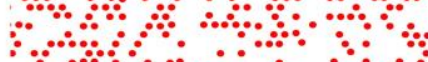
$\because \therefore \therefore$ : $\because \cdots$

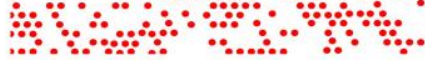
$<\operatorname{lmn}>$ 
Figure 4

a

C

Radar Plots for a(W-W) of W0.44Mo0.56S2

Growth direction

in the region of interest

$1 / 3<11-2>$

1 region of interst

Mox $W_{1-x} S_{2}$ flake

(1)
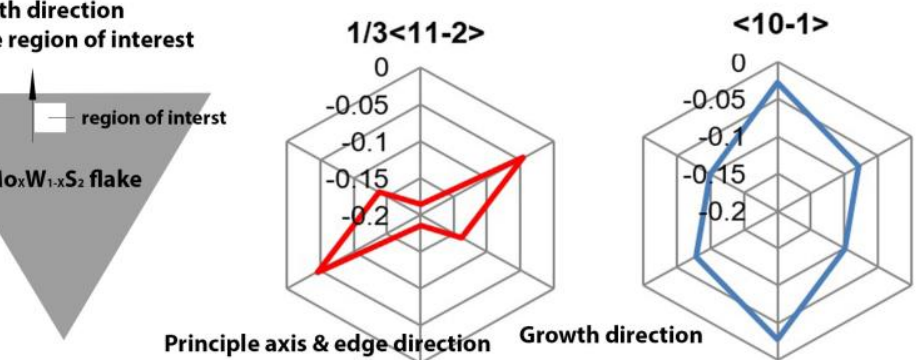

Principle axis \& edge direction Growth direction

b
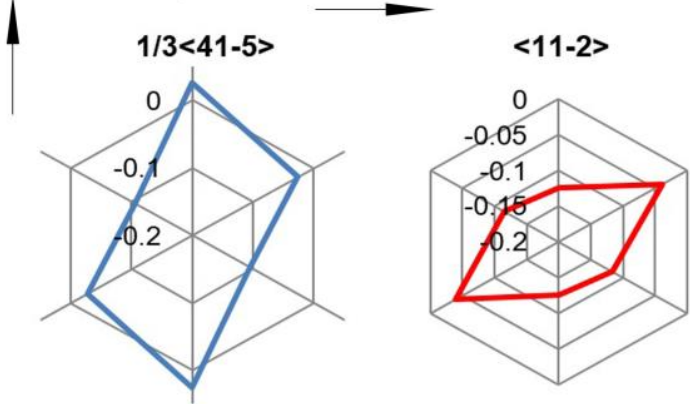

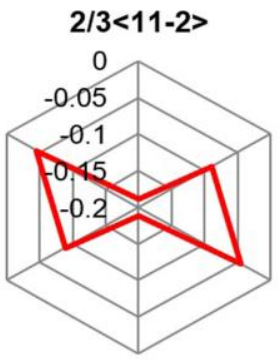

$4 / 3<11-2>$

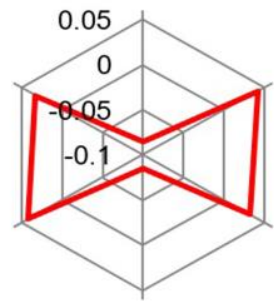




\section{Figure 5}
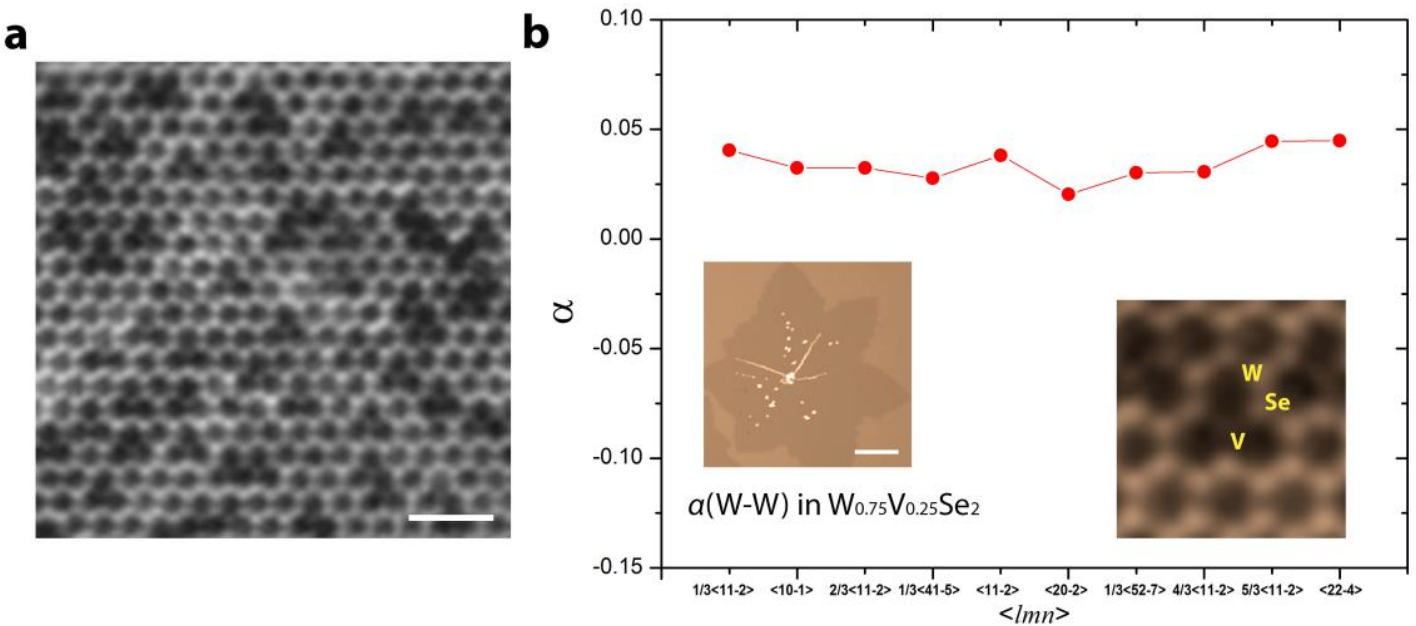